\title{
Selective deposition of polycrystalline silicon thin films at low temperature by hot-wire chemical vapor deposition
}

\author{
S. Yu, ${ }^{\text {a) }}$ E. Gulari, ${ }^{\mathrm{a}}$ and J. Kanicki ${ }^{\mathrm{b})}$ \\ Center for Display Technology and Manufacturing, University of Michigan, Ann Arbor, Michigan 48109
}

(Received 19 December 1995; accepted for publication 1 March 1996)

\begin{abstract}
Polycrystalline silicon thin films have been selectively deposited at a substrate temperature of $300{ }^{\circ} \mathrm{C}$ on molybdenum or silicon over silicon dioxide, silicon nitride or Corning 7059 glass substrates in a continuous hot-wire chemical vapor deposition (HWCVD) process involving hydrogen and disilane. Excellent selectivity is achieved on features as small as $1 \mu \mathrm{m}$ spaced molybdenum lines. The deposition rate on molybdenum is $60-100 \AA / \mathrm{min}$ which is higher than that obtained by a pulsed gas plasma-enhanced chemical vapor deposition (PECVD) or a very high-frequency PECVD (VHF-PECVD). The selective deposition rate obtained in our system is attributed to the high concentration of atomic hydrogen generated when molecular hydrogen passes over a heated tungsten filament. (C) 1996 American Institute of Physics.
\end{abstract}

[S0003-6951(96)01619-1]

Selective deposition of polycrystalline silicon (poly-Si) thin films has important applications in fabrication of hydrogenated amorphous silicon $(a-\mathrm{Si}: \mathrm{H})$ thin-film transistors (TFTs) for liquid crystal displays. For example, in top- or bottom-gate TFT configuration, by selective depositing source and drain contact layers the $a$-Si:H TFT array fabrication process can be simplified. This simplification will provide a path to low cost TFT array manufacturing and will extend present $a-\mathrm{Si}: \mathrm{H}$ technology to a very high resolution, large area displays. Selective deposition of microcrystalline silicon thin films has been achieved by a pulsed gas PECVD ${ }^{1}$ or a very high-frequency plasma-enhanced chemical vapor deposition (VHF-PECVD) process ${ }^{2}$ using $\mathrm{SiH}_{4}$ and $\mathrm{H}_{2}$ as reactants or a conventional rf-plasma CVD using $\mathrm{SiH}_{4}$ and $\mathrm{SiF}_{4}$ mixtures. ${ }^{3}$ However, the growth rates of the selectively deposited films by these methods are low and/or a substrate temperature higher than $300{ }^{\circ} \mathrm{C}$ is required. In this letter, we have demonstrated that selective deposition of polycrystalline silicon can be achieved by hot-wire chemical vapor deposition (HWCVD) at a growth rate of $100 \AA / \mathrm{min}$ and a substrate temperature of $300{ }^{\circ} \mathrm{C}$ with excellent selectivity over metal lines spaced $1 \mu \mathrm{m}$ apart.

HWCVD employs a heated tungsten filament instead of a plasma to activate reactive gases. Its gas phase chemistry is simpler than that of PECVD, there are no film damages caused by bombardment of energetic species, and it is cost effective for large area applications. A variety of good quality materials such as amorphous silicon and silicon nitride have been deposited at high growth rates by this method. ${ }^{4,5}$ The HWCVD reactor employed in this study has been described in some detail in previous papers. ${ }^{6,7}$ In brief, the reactor consists of a cold wall 6 in. six-way stainless-steel chamber. The reactor has a quartz viewport through which the filament temperature is monitored with an optical pyrometer. The filament unit consists of a tungsten filament of about $1.0 \mathrm{~m}$ in length and $0.25 \mathrm{~mm}$ in diameter wrapped around two quartz rods. Substrates of 2 in. in diameter are

\footnotetext{
a) Also with the Department of Chemical Engineering.

${ }^{b)}$ Also with the Department of Electrical Engineering and Computer Science.
}

clamped to a stainless-steel susceptor which is heated by two inserted cartridge heaters. The substrate temperature is monitored with a thermocouple clamped to the susceptor surface close to the substrate. The distance between the filament and the substrate is $4 \mathrm{~cm}$. We pass hydrogen gas over the surface of the heated filament where it is catalytically disassociated into atomic hydrogen. Unlike the HWCVD process used by other groups, ${ }^{4,8}$ we flow source gas disilane through a dispersal ring bypassing the filament and let it react with atomic hydrogen downstream of the filament to form film forming species. In this way, we avoid both the generation of atomic Si which has a high sticking coefficient undesirable for good quality films and the deterioration of the tungsten filament when silicon atoms react with tungsten to form tungsten silicides. The formation of tungsten silicides will also reduce the efficiency of the tungsten wire to produce atomic hydrogen needed for a high rate selective deposition process. Using the process described above, we have deposited poly-Si films at $300{ }^{\circ} \mathrm{C}$ on glass substrates with certain hydrogen to disilane ratio. ${ }^{7}$

In this study, poly-Si thin films are deposited at a substrate temperature of $300{ }^{\circ} \mathrm{C}$, hydrogen flow rate of $48-97$ sccm, disilane flow rate of 0.53-1.6 sccm, pressure of 40-65 mTorr, and filament temperature of $1800^{\circ} \mathrm{C}$. Silicon content in the gas phase is varied by adjusting the flow rate of hydrogen or disilane. Molybdenum is deposited by sputtering and then patterned by photolithography or by using a mechanical mask to cover part of the substrate during sputtering. Under these conditions, poly-Si thin films have been selectively deposited on molybdenum, amorphous, polycrystalline, and single-crystal silicon over thermal or PECVD silicon dioxide, silicon nitride and Corning 7059 glass substrates. Selective deposition on indium tin oxide (ITO), however, is difficult to achieve in our system, since reduction of ITO by the atomic hydrogen present in the process occurs before film growth stars. The film thickness and crystallinity are examined by cross-section transmission electron microscopy (TEM). The deposition rate is calculated by dividing the film thickness by the total process time. The selectively deposited films on patterned substrates are examined by scanning electron microscopy (SEM). 


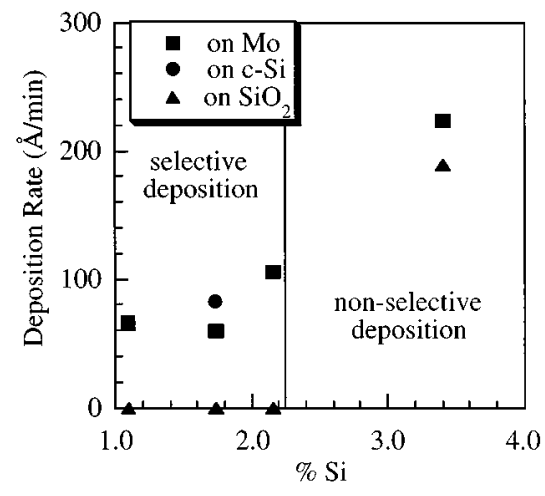

FIG. 1. Dependence of deposition rate and selectivity on Si content in the gas phase.

The dependence of deposition rate and selectivity on Mo and $c$-Si over $\mathrm{SiO}_{2}$ at a substrate temperature of $300{ }^{\circ} \mathrm{C}$ on $\mathrm{Si}$ content in the gas phase is shown in Fig. 1. Selective deposition is obtained at $\mathrm{Si}$ content below $2.2 \%$ in the gas phase. At higher Si content in the gas phase, selectively is lost and films are deposited on both $\mathrm{Mo}$ and $\mathrm{SiO}_{2}$ with the net deposition rate on Mo higher than on $\mathrm{SiO}_{2}$. The selective deposition rate on Mo or $c$-Si over $\mathrm{SiO}_{2}$ shown in Fig. 1 is higher than the rate obtained by the pulsed gas $\mathrm{PECVD}^{1}$ or VHF-PECVD ${ }^{2}$ or the rf-plasma CVD using $\mathrm{SiH}_{4}$ and $\mathrm{SiF}_{4}$ mixtures. ${ }^{3}$ Under the selective deposition conditions, we have observed that $a-\mathrm{Si}: \mathrm{H}$ is etched at a rate at least one order of magnitude faster than the etching rate observed in a VHF-PECVD system. This indicates that the concentration of atomic hydrogen in our system is much higher than that in a VHF-PECVD system, which implies that catalytic disassociation of molecular hydrogen by a heated tungsten filament is a much more efficient way than a plasma in generating atomic hydrogen.

The generation rate of atomic hydrogen increases with filament temperature and hydrogen pressure. At higher filament temperatures contamination by tungsten will become a problem. However, at a filament temperature of below $2100{ }^{\circ} \mathrm{C}$, tungsten level in a HWCVD silicon film is at the detection limit of secondary ion mass spectroscopy. ${ }^{9}$ Therefore, at a filament temperature of $1800{ }^{\circ} \mathrm{C}$ used in this study, tungsten contamination should not be a concern.

The TEM cross-section micrograph with the selected area electron diffraction pattern of a film selectively deposited on $\mathrm{Mo}$ over $\mathrm{SiO}_{2}$ at a $\mathrm{Si}$ content of $1.1 \%$ in the gas phase is shown in Fig. 2. The TEM micrograph as well as the selected area diffraction pattern shows that the film is nontextured poly-Si with randomly oriented crystallites. When they are examined by cross-section TEM, the poly-Si films nonselectively deposited on both $\mathrm{Mo}$ and $\mathrm{SiO}_{2}$ at higher $\mathrm{Si}$ content in the gas phase exhibit similar microstructure and crystallinity.

The SEM micrographs of patterned Mo lines of $1720 \AA$ thick with a spacing of $1 \mu \mathrm{m}$ on $\mathrm{SiO}_{2}$ before and after selective deposition are shown in Fig. 3. It can be seen that the poly-Si film is deposited on $1 \mu \mathrm{m}$ spaced Mo lines but not on $\mathrm{SiO}_{2}$. By cross-section SEM of this sample, we have ob-

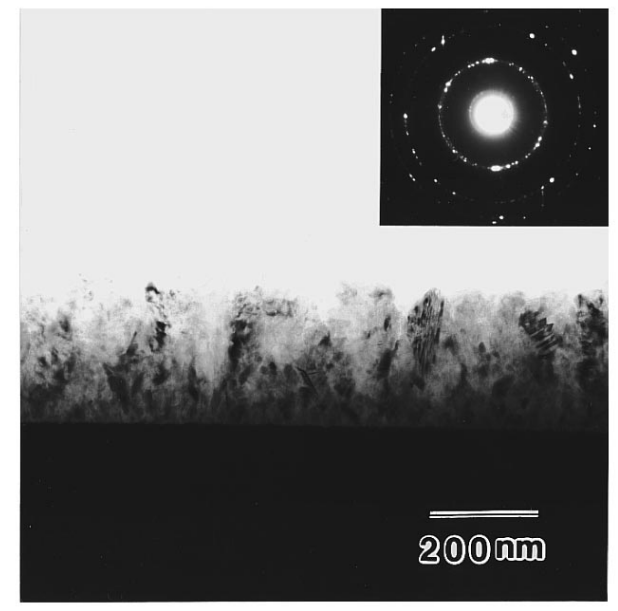

FIG. 2. TEM cross-section micrograph with selected area diffraction pattern of a film selectively deposited on $\mathrm{Mo}$ over $\mathrm{SiO}_{2}$ at $1.1 \% \mathrm{Si}$ showing that the film is nontextured polysilicon.

served that the poly-Si film is also deposited on the side walls of the Mo lines. The experimental conditions for this deposition are the same as the conditions used for the film whose TEM micrograph is shown in Fig. 2. Therefore, the film is polycrystalline. In addition, by comparing the thickness of Mo lines before and after deposition from crosssection SEM, the thickness of the film is $\sim 1200 \AA$ for a 20 min deposition process. This gives a deposition rate of 60 $\AA /$ min, which is very close to that measured by TEM of the

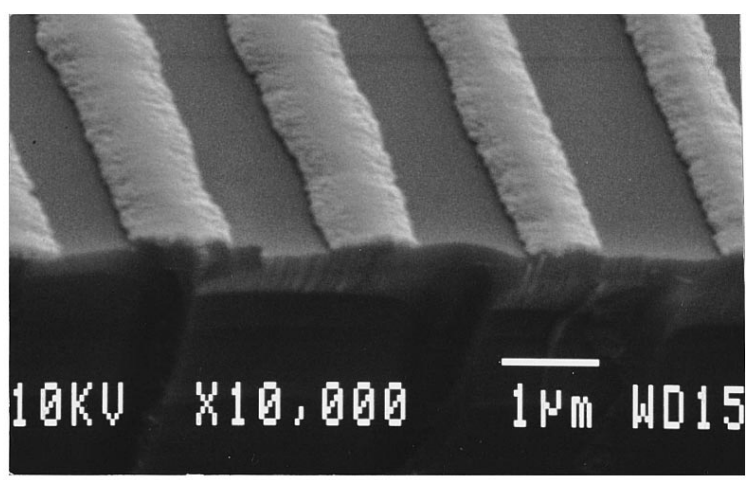

(a)

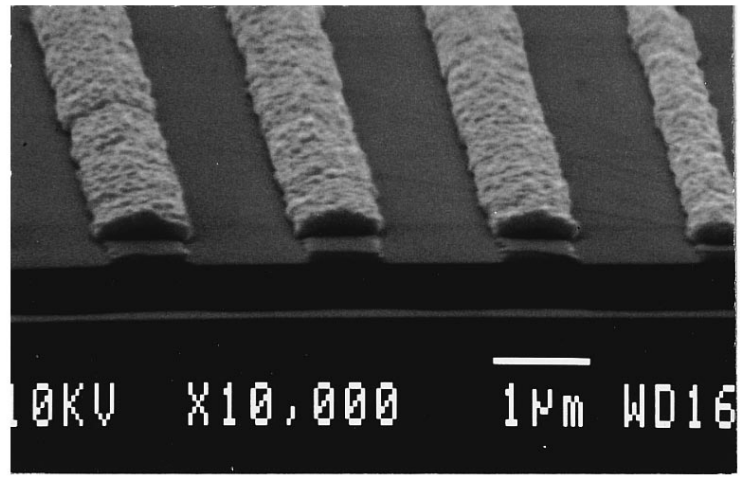

(b)

FIG. 3. SEM micrographs of $1 \mu \mathrm{m}$ spaced Mo lines over $\mathrm{SiO}_{2}$ before deposition (a), and after deposition (b). 
film selectively deposited under the same condition on Mo over $\mathrm{SiO}_{2}$ of a much larger area.

It has been found that atomic hydrogen plays a key role in substrate selective deposition. ${ }^{2,10}$ The net growth rate of the selective deposition is a result of the difference of a silicon deposition rate and etching rate of strained $\mathrm{Si}-\mathrm{Si}$ bonds by atomic hydrogen. The substrate selectivity is attributed to the difference of nucleation of $\mathrm{Si}-\mathrm{Si}$ bond strength on different substrates. However, the origin of the difference of nucleation on different substrates is not pinpointed. Recently, Roca i Cabarrocas et al. have suggested $a$-Si:H nucleation versus homogeneous growth mechanisms to explain the substrate selectivity. ${ }^{11}$ However, contradictory to the homogeneous growth on $a-\mathrm{Si}: \mathrm{H}$ observed by Roca i Cabarrocas et $a l .,{ }^{11}$ we have deposited poly-Si selectively on $a$-Si:H substrate that has been etched during the selective deposition process. Etching of $a$-Si:H substrate is in agreement with the mechanism that etching by atomic hydrogen takes place during the initial nucleation process. However, this mechanism does not provide an explanation for the difference of nucleation on different substrates. In our opinion, the microscopic mechanism of substrate selectivity still remains unclear and further investigation is needed.

${ }^{1}$ G. N. Parsons, Appl. Phys. Lett. 59, 2546 (1991).

${ }^{2}$ W. Westlake and M. Heintze, J. Appl. Phys. 77, 879 (1995).

${ }^{3}$ K. Baert, P. Deschepper, J. Nijs, and R. Mertens, Mater. Res. Soc. Symp. Proc. 164, 395 (1989).

${ }^{4}$ A. H. Mahan, J. Carapella, B. P. Nelson, and R. S. Crandall, J. Appl. Phys. 69, 6728 (1991).

${ }^{5}$ S. V. Deshpande, J. L. Dupuie, and E. Gulari, Appl. Phys. Lett. 61, 1420 (1992).

${ }^{6}$ J. L. Dupuie and E. Gulari, J. Vac. Sci. Technol. A 10, 18 (1992).

${ }^{7}$ S. Yu, S. V. Deshpande, E. Gulari, and J. Kanicki, Mater. Res. Soc. Symp. Proc. 377, 69 (1995).

${ }^{8}$ H. Matsumura, Y. Tashiro, K. Sasaki, and S. Furukawa, Jpn. J. Appl. Phys. 33, L1209 (1994)

${ }^{9}$ C. Horbach, W. Beyer, and H. Wagner, J. Non-Cryst. Solids 137\&138, 661 (1991).

${ }^{10}$ J. J. Boland and G. N. Parsons, Science 256, 1304 (1992).

${ }^{11}$ P. Roca i Cabarrocas, N. Layadi, T. Heitz, and B. Drevillon, Appl. Phys. Lett. 66, 3609 (1995). 